\title{
LA VIDA AFECTIVA EN LA TEORÍA DE LA EDUCACIÓN. EL TRATAMIENTO DE LAS EMOCIONES EN EL SEMINARIO INTERUNIVERSITARIO DE TEORÍA DE LA EDUCACIÓN*
}

\author{
The affective life in Educational Theory. The treatment \\ of emotions in the Seminario Interuniversitario de Teoría \\ de la Educación
}

\section{La vie affective dans la Théorie de l'Éducation. Le traitement des émotions dans le Seminario Interuniversitario de Teoría de la Educación}

\begin{abstract}
Miriam PRIETO EGIDO
Universidad Complutense de Madrid. Facultad de Educación. Departamento de Teoría e Historia de la Educación. c/ Rector Royo Villanova, s/n. 28040 Madrid. Correo-e: miriampr@edu.ucm.es
\end{abstract}

Fecha de recepción: enero de 2011

Fecha de aceptación definitiva: abril de 2011

Biblid [(1130-3743) 23, 1-2011, 107-129]

* El presente trabajo forma parte de una tesis doctoral financiada por el Programa de Formación de Profesorado Universitario (FPu) del Ministerio de Educación, que pretende ahondar en el papel del miedo -considerado en el marco más amplio de las emociones- en el ámbito educativo. Asimismo, se inscribe en la línea de investigación "Procesos de modernización en la educación: el sistema educativo en el contexto de la edad secular y la cultura biopolítica" del Grupo de Investigación Validado UCMCAM: Cultura Cívica y Políticas Educativas (Referencia: 930768), de la Facultad de Educación de la Universidad Complutense de Madrid. 


\section{RESUMEN}

¿Qué lugar han ocupado las emociones en la definición de ser humano sobre la que se ha construido la Teoría de la Educación española? ¿Qué reconocimiento se ha otorgado al sistema afectivo en la reflexión acerca de lo que es o ha de ser la educación? Éstas son las dos preguntas que el presente artículo pretende responder. Para ello se presenta una revisión del trabajo realizado en el seno del Seminario Interuniversitario de Teoría de la Educación, dado el papel destacado que éste ha desempeñado en la configuración de la Teoría de la Educación en nuestro país. Las respuestas a estas cuestiones vienen de la voz de los propios miembros del siTE, cuyas reflexiones a este respecto se presentan de manera resumida pero detallada, de manera que el propio lector pueda seguir su evolución.

Palabras clave: teoría de la educación, afectividad, ser humano, educación.

\section{SUMMARY}

What place have emotions taken in the definition of human being on which the Spanish Educational Theory has been built? What recognition has been given to the affective system in reflections about what is or must be education? These are both questions that the present article tries to answer. To do so, a review of the work that has been made within the Seminario Interuniversitario de Teoria de la Educación is presented, considering the important role it has played in shaping the Theory of Education in Spain. The answers to these questions come from the members of the seminar themselves, whose reflections in this regard are presented in a summarized but detailed way, in such way that the own reader is able to follow this development.

Key words: educational theory, feeling, human being, education.

\section{SOMMAIRE}

Quel lieu ont-ils occupé les émotions dans la définition de l'être humain sur laquelle on a construit la Théorie de l'Éducation espagnole? Quelle reconnaissance a-t-elle été accordée à l'appareil affectif dans la réflexion à propos de ce qu'elle est ou doit être l'éducation? Ce sont les deux questions auxquelles l'article présent essaie de répondre. Pour cela, on se présente une révision du travail developpé au sein du Seminario Interuniversitario de Teoría de la Educación, compte tenu du rôle éminent qu'il a joué dans la configuration de la Théorie de l'Éducation à l'Espagne. Les réponses à ces questions viennent de la voix des propes membres du siTE, dont les réflexions à ce sujet se présentent d'une manière résumée mais détaillée, pour que le prope lecteur puisse suivre son évolution.

Mots clés: théorie de l'éducation, affectivité, être humain, éducation. 


\section{INTRODUCCIÓN}

Pese a lo que se señala al definir al ser humano como ser "racional", no es sólo la racionalidad, sino también la afectividad, lo que caracteriza al ser humano y lo diferencia del resto de animales. Desde los griegos lo sabemos: es por nuestra capacidad de sentir - de sufrir- por lo que nos diferenciamos de los gorriones. El lugar de una y otra, la preponderancia de la razón o de la pasión, su interrelación o dependencia, están por determinar, y han sido tema de disputa incesante en la historia del pensamiento. Sin embargo, hemos de aceptar que el hombre no es sólo el ser que no puede sino pensar, sino también el sujeto que, inevitablemente, siente. Partiendo de esta premisa, el presente trabajo pretende, dicho con palabras de J. García Carrasco, "situar la afectividad y la emocionalidad en un lugar armónico y no por mera yuxtaposición dentro de la totalidad del discurso" educativo (García Carrasco y Núñez Cubero, 1995, 152). Para ello, más que derivar una serie de argumentos personales, interesa ahora rastrear el estatuto de las emociones en la teoría educativa. En este sentido, este trabajo se centra en el análisis de la consideración que se ha otorgado a las emociones en la Teoría de la Educación española en las últimas décadas.

Para ello, se han seleccionado las ponencias presentadas en el Seminario Interuniversitario de Teoría de la Educación (SITE) ${ }^{1}$, desde su fundación en 1982, hasta la actualidad. Si bien el conocimiento pedagógico elaborado en este espacio no agota ni recoge todo el trabajo realizado en el ámbito de la Teoría de la Educación española, sí constituye una muestra representativa de las principales tendencias, paradigmas y orientaciones desde la década de los 80, años en los que "se desarrolla una consolidación de la disciplina como conocimiento científico y tecnológico" (Naval, 2008, 38), hasta hoy. Dado que la creación del site data de años recientes, la información obtenida en la revisión documental se ha completado y contrastado con entrevistas a algunos de los profesores que han participado en él

1. El Seminario Interuniversitario de Teoría de la Educación se funda en 1982 en la Universidad de Murcia, en una reunión a la que asistieron los profesores Alexandre Sanvisens, de la Universidad de Barcelona; José Antonio Ibáñez Martín y Gonzalo Vázquez, de la Universidad Complutense de Madrid; José María Quintana, de la unED; José Luis Castillejo, de la Universidad de Valencia; Joaquín García Carrasco, de la Universidad de Salamanca; Jaume Sarramona, de la Universidad Autónoma de Barcelona; Antoni Colom, de la Universidad de las Islas Baleares, y Juan Escámez, de la Universidad de Murcia. El seminario consiste en "una reunión a puerta cerrada, de carácter anual, que congrega a los expertos de la sub-área universitaria de Teoría de la Educación de la Universidad española, con el objeto de discutir y analizar en profundidad una serie de trabajos o ponencias marco, que por lo general poseen carácter definidor y fundamentante, a fin de introducir en la comunidad científica nuevas aportaciones, nuevas perspectivas o enfoques". Colom (1994, 10). 
en distintos momentos de su historia, tomando como criterio para su selección su trayectoria en el estudio del sistema emocional o de la Teoría de la Educación ${ }^{2}$.

Así, en este trabajo se mostrará el recorrido que han seguido las alusiones y consideraciones del sistema emocional en las ponencias del Seminario, lo que responde, a su vez, a una doble intención: que el propio lector pueda seguir el camino encontrado en el análisis de las fuentes, por un lado, y mostrar el aumento progresivo del interés acerca de la relación entre afectividad y educación que se ha ido cuajando en los trabajos del Seminario, por otro. Estas menciones se presentan en relación con dos categorías: la idea de ser humano y la concepción de la educación. "En el planteamiento de los fines desempeñan un papel decisivo una serie de creencias previas - políticas, sociales, religiosas, etc.- como también un número de ideas básicas: la idea de hombre, su puesto en la sociedad, su trascendentalidad, etc. Queramos o no, todos estos factores intervienen en la definición y, en último término, en la concepción de la acción educativa" (Ferrández y Sarramona, 1984, 18). Por ello, se ha considerado pertinente en este análisis partir de la idea de ser humano sobre la que se construye el discurso educativo en relación con la emocionalidad, así como la consideración de los elementos pasionales en el concepto de educación que se ha ido construyendo en el seno del Seminario ${ }^{3}$.

\section{PRIMER PERIODO: EL SILENCIO EMOCIONAL}

El primer seminario, celebrado en 1982, llevaba por título "La teoría de la educación: líneas de investigación y ámbitos de aplicación", que fue seguido de "Teoría de la educación. (El problema de la educación I)" (AA.vV., 1983). Estos dos seminarios sirvieron para "consolidar el grupo y definir la dinámica del propio seminario" (Colom, 2003, 148). En los seminarios ulteriores ${ }^{4}$, se aprecia una clara intención de hacer de los procesos educativos un conocimiento científicotécnico. Así lo resume J. Escámez (1986), quien en su ponencia presentada en el SITE de 1985 afirma que el pedagogo es un tecnólogo, cuya actividad se centra en la racionalidad.

2. Agradezco la colaboración de los profesores O. Fullat, uno de los primeros en destacar el componente pasional del ser humano; J. García Carrasco, el primero en presentar una ponencia sobre el aparato emocional en el Seminario; M. Martínez y G. Jover, quienes participan activamente en la organización del Seminario en el momento actual. La información y experiencia aportada por estos profesores ha sido crucial para la interpretación de las fuentes.

3. Como aclaración metodológica, señalar que para este trabajo se ha dejado al margen la discusión acerca de las diferencias entre los diversos términos que refieren la vida emocional (sentimientos, emociones, afectos, etc.), ya que lo que interesaba era recopilar las referencias a la vida emocional en su conjunto.

4. Nos referimos, por orden cronológico, a las siguientes publicaciones: AA.vv. (1985 y 1986); SARRAMONA (1987 y 1988). 


\subsection{La idea de ser humano}

¿Quién es el ser humano en esta Teoría de la Educación científico-técnica? Podemos afirmar que durante este primer periodo predomina la concepción del hombre como ser racional. Sin embargo, encontramos algunas voces que nos recuerdan que el ser humano es eso, pero también algo más. Así, J. A. IbáñezMartín, en el SITE celebrado en 1986 bajo la temática Curriculum y Educación, finaliza su ponencia afirmando:

Voy a terminar sumándome a las ideas de Martin, cuando pide un nuevo paradigma en la construcción del currículo, un paradigma que «integre el pensamiento y la acción, la razón y la emoción, la educación y la vida: uno que no divorcie a las personas de su contexto social y natural; uno que abrace la autonomía individual pero como uno de los muchos valores" (Ibáñez-Martín, 1987, 22).

En este mismo Seminario, el profesor O. Fullat, en una ponencia que versa sobre la "Fundamentación política del currículum", defiende que "el hombre no es, de entrada, animal racional -esto constituye una hermosa aspiración, pero poco más-, sino que se comporta como animal de pasiones. Predomina en nuestra especie el pathos por encima del logos" (Fullat, 1987, 49).

Un año después, serán los profesores R. Medina y T. Rodríguez quienes, al hablar de la relación entre comunicación y educación, definan al sujeto como "aquellos seres capaces de sentir, desear, apetecer, imaginar, recordar, pensar, razonar y captar, directa, inmediata y conscientemente cada uno de sus actos" (Medina y Rodríguez, 1988, 27).

El profesor R. Marín, en el site de 1988, celebrado en torno a la Educación Social, aborda los ámbitos esenciales de la formación cívica, identificando entre ellos el análisis de las bases éticas de la convivencia social, ámbito que considera uno de los más necesitados de profunda reflexión. Ésta debe partir de la constatación de un hecho elemental: "La convivencia social no es un fenómeno físico -que se dé al margen de nuestros deseos- sino una realidad moral, que se pone en existencia como consecuencia de la decisión de las personas y que tiene bases tanto antropológicas como, específicamente, éticas. No será ocioso recordar las habilidades y sentimientos sociales que el hombre posee" (Marín, 1988, 408).

Vemos que ya en la década de los ochenta en España algunas voces recuerdan y reclaman una visión más holística y completa del ser humano que incluye al sistema emocional en su definición. Sin embargo, apreciamos que estas menciones no son sólo escasas en cantidad, sino que se dan desligadas de una reflexión o análisis de las implicaciones del sistema emocional en el ser humano.

Entramos ya en la década de los noventa, durante la cual se irá gestando un cambio en la concepción teleológica de la educación, que se reflejará también en la consideración que se otorga a las emociones. Sin embargo, durante los primeros años de esta década se mantiene la trayectoria seguida en la década anterior, y también las escasas referencias a las emociones. 
La primera mención a la afectividad en relación al ser humano de esta década la encontramos en el siTe celebrado en 1991 en torno a la educación no formal. Los profesores J. Escámez y P. M. Alonso-Geta destacan como elemento esencial de la planificación la acción comunicativa, en la que, a su vez, otorgan un lugar destacado al acuerdo. Si bien afirman que éste "descansa sobre una convicción común y sólo se alcanza bajo condiciones que conllevan una base racional", identifican como partes constituyentes de tal acuerdo el mundo objetivo y el mundo subjetivo, compuesto este último por las intenciones, deseos y sentimientos personales (Escámez y Alonso-Geta, 1992, 80).

Dos años más tarde, en el site celebrado en 1993 y que tuvo por título Política y planificación educativa, el profesor A. J. Colom señala que planificar la educación requiere conocer al ser humano, "abarcar una comprensión general de la estructura del sistema así como del funcionamiento de sus elementos básicos -roles, creencias, sentimientos, expectativas, metas, objetivos, normas, redes de poder e influencia, sanciones..., etc.- para luego poder proyectar futuras situaciones hipotéticas pero realmente asumibles" (Colom, 1994, 168).

Este mismo año, los profesores F. Etxeberría, J. Trilla y X. Úcar presentan una ponencia sobre la educación no formal y las políticas culturales. En este marco abordan diversas acepciones del término "cultura", para finalmente concluir que la cultura "es prácticamente el equivalente a "lo humano" (Etxeberría et al., 1992, 246). Así, ésta sería "el conjunto de rasgos distintivos, espirituales y materiales, intelectuales y afectivos que caracterizan a una sociedad o grupo social. Engloba, además, las artes y las letras, los modos de vida, los derechos fundamentales del ser humano, los sistemas de valores, las tradiciones y las creencias" (Etxeberría et al., 1992, 246).

\subsection{Consideraciones sobre la educación}

¿Qué idea de educación se articula en estos años? ¿Qué consideración de las emociones entraña?

La educación es, o puede pensarse como, acción y, si vale la redundancia, entre lo racional y el ser-pensado, cabe pensarse como acción racional. Los referidos estudios de la acción educativa, hechos desde las perspectivas teorética, tecnológica, sociológica cibernética -entre otras- así lo muestran. Características de la acción educativa como acción racional serían las de internacionalidad, propositividad, teleología, equifinalidad, intervención, contextualidad, normatividad, eficacia, eficiencia y valiosidad (Vázquez Gómez, 1987, 74).

La educación se entiende como una actividad orientada al desarrollo de la racionalidad, de la inteligencia y el conocimiento, esto es, de la capacidad cognitiva, y al aprendizaje desde una perspectiva instrumental. La persona educada es, por tanto, la persona con niveles crecientes de racionalidad (Escámez, 1983). 
Durante este periodo encontramos también algunas referencias a la importancia de la educación moral. Así, J. Escámez, en su ponencia "Marginación socioeconómica y educación" presentada en el IIISITE84, afirma que "podemos considerar como educación la consecución por el educando, a través de un proceso de intervención, de un estado deseable considerado como patrón alcanzado; este estado deseable implicaría el desarrollo del conocimiento y la inteligencia, formando esquemas conceptuales propios, así como la incorporación práctica, o apropiación personal, de valores morales rectores del comportamiento" (Escámez, 1985, 115).

Si bien en la consideración de la idea de hombre que se maneja durante este periodo encontramos algunas voces discordantes con el modelo cognitivo predominante, no sucede lo mismo en el caso de la educación. Las menciones que se hacen a las emociones son escasas y siempre orientadas hacia el fin prioritario, el aprendizaje, entendiéndose éstas como un medio, un elemento clave de la disponibilidad para aprender, "en el sentido de que en ausencia de emocionalidad (en sentido amplio) no se promueven actividades de procesamiento" (Castillejo, 1988, 49).

Sí se hace alguna mención de las emociones en el ámbito educativo al abordar la relación educativa entre educador y educando. "La acción educativa se hace posible cuando la relación docente-discente es positiva y auténtica. Se trata de una relación profunda que se orienta a la mejora del mundo personal, intelectual y afectivo, del educando" (López-Barajas, 1988, 65). También se afirma que "la aceptación del educando al educador depende del grado de adaptación conceptual, y también, en todo caso, afectiva entre uno y otro" (Sanvicens, 1985, 85). Vemos, sin embargo, que predomina la tradicional jerarquización de la razón sobre la pasión.

\section{SEgundo PERIOdO: LA ECLOSIÓN EMOCIONAL}

Mediados los años 90 asistimos a la gestación que hemos denominado «eclosión emocional" y que alcanzará su máxima expresión en la década de los años 2000. Lo que en años anteriores eran menciones aisladas y puntuales a las emociones se convierten a partir de este momento en referencias que proliferan y se mantienen como una constante entre la variabilidad de aspectos de la educación abordados, hasta el punto de ser protagonistas en el año 2006.

¿Cuál es el contexto de esta eclosión? En estos años se produce en el Seminario un cambio organizativo y metodológico que conlleva la apertura a nuevas voces que recogen los ecos de los organismos internacionales, y que traen de la mano el traslado del acento de la tecnología a la reflexión y de los valores generales al bienestar individual, con el consecuente acento en los aspectos subjetivos, entre ellos, el sistema emocional. A estos ecos internacionales hemos de añadir nuevas corrientes en relación con las emociones que se gestan en diversos ámbitos de conocimiento, especialmente en dos: la psicología y la neurociencia. En el primero asistimos en 1996 a la traducción al español del libro de D. Goleman Emotional Intelligence, que había sido publicado un año antes. En el ámbito de la neurociencia, también en 1996 se traduce el libro del portugués A. Damasio Descartes' error: emotion, 
reason and the buman brain, publicado en 1994, en el que el autor explora la relación entre las emociones, los sentimientos y sus bases neurológicas partiendo de la crítica a la escisión entre mente y cuerpo. Estas voces resuenan en el interior del Seminario, lo que puede comprobarse en la bibliografía referida cuando se menciona o aborda el componente emocional, protagonizada en la mayoría de los casos por D. Goleman o A. Damasio, cuando no ambos. Veamos, pues, la forma y el contenido que adquiere este nuevo interés en el interior del sITE.

\title{
2.1. La idea de ser humano
}

Si bien hasta este momento las referencias al sistema afectivo son escasas, concretas y particulares, en el año 1994 asistimos a una primera "eclosión" del sistema emocional. La primera ponencia presentada en este Seminario, de la mano de O. Fullat y J. C. Mèlich, hace hincapié en los límites del ser humano como ser racional. Estos autores señalan los límites de la razón a la hora de la fundamentación de los valores. "La razón no obliga porque no se dirige a la libertad, sino al entendimiento y éste se limita a entender. Ninguna necesidad práctica proviene de la razón. El imperativo a la voluntad no puede deducirse de la razón práctica. La razón entiende pero jamás manda" (Fullat y Mèlich, 1995, 31). Unas páginas más adelante caracterizarán la cultura occidental como "el intento de acabar con todo lenguaje "no lógico" (1995, 59). Frente al logos aristotélico y platónico, reivindican la ruptura que Nietzsche supone de la ecuación aristotélica "razón = virtud = felicidad" (1995, 59). Continúan con una cita de Nietzsche:

\begin{abstract}
El moralismo de los filósofos griegos a partir de Platón tiene unos condicionamientos patológicos; y lo mismo su aprecio a la dialéctica. Razón=virtud=felicidad significa simplemente: hay que imitar a Sócrates e implantar de manera permanente, contra los apetitos oscuros, una luz diurna; la luz diurna de la razón. Hay que ser inteligentes, claros, lúcidos a cualquier precio: toda concesión a los instintos, a lo inconsciente, conduce hacia abajo $(1995,59)$.
\end{abstract}

Tras esta apertura del Seminario, los profesores F. Etxebarría y J. Sarramona, coordinados por J. A. Jordán, abordan la noción de «identidad cultural», que convienen en definir como "lo que uno es (preferencias, comportamientos, valores...) en virtud de su adhesión afectiva y/o funcional a una comunidad cultural (o más), que proporciona una forma de "estar-en-el-mundo" susceptible de interpretar la realidad de modo diferenciado y, a la vez, uniforme". La identidad sería "el hacer, sentir y pensar de un sujeto" (Etxebarría et al., 1995, 100).

Cierran este Seminario los profesores J. L. Núñez Cubero y A. Caride, coordinados por J. García Carrasco, con la ponencia titulada "Educación afectiva y de la sensibilidad" (Caride et al., 1995). Las emociones ocupan un lugar destacado en las relaciones que el ser humano establece con el entorno, y las funciones de la sensibilidad en la orientación, la atención y la valoración del sí mismo. La complejidad del mundo hace que la capacidad intelectual-lógica sea insuficiente para evaluar e 
integrar lo que percibimos. En esta evaluación son de gran utilidad los sentimientos, pues son éstos un elemento esencial en la vivencia de la armonía personal, esto es, el equilibrio entre deseos y realidad. El trabajo que estos profesores presentan pretende subrayar la importancia de la afectividad en la modelación del comportamiento. Defienden que el placer de la satisfacción y el apego son sucesos biológicos y sistemas de comportamientos psíquicos primarios; es decir, en ellos se hace patente la unidad psicosomática del ser humano y su presencia se puede rastrear en fenómenos sociales tales como la amistad, la fidelidad, la gratitud, la identificación con un modelo, el apego a un lugar, y en la construcción de hábitos y costumbres. Así, el apego sería la base para la construcción del resto de mecanismos afectivos.

Al año siguiente, en 1995, asistimos a la temática Acción educativa y comunicación social. Los profesores J. M. Esteve, J. M Puig y T. Romañá se refieren a «La educación en los grupos primarios". "Los grupos primarios son espacios sociales en los que la intensidad de las relaciones y los lazos afectivos que unen a sus miembros los hacen muy proclives a producir intensos efectos educativos" (Esteve et al., 1996, 72), entre los que se pueden citar la construcción de la identidad personal, la transmisión de valores, la adquisición de patrones conductuales, motivos y fines para la acción, destrezas sociales y desarrollo de capacidades psicológicas básicas. Sitúan por tanto el sistema emocional en la base del desarrollo intelectual, social y moral.

No volveremos a encontrar referencias a la concepción del ser humano en relación a las emociones hasta 1999, año en que el Seminario se reúne en torno a La educación obligatoria. Competencias básicas del alumno. Los profesores J. Vera, A. García del Dujo, P. Calvo y B. Gargallo trabajan sobre los criterios de selección del currículum. Entre las fuentes que intervienen en ese proceso de selección identifican la herencia cultural, que definen como "un repertorio evolutivo de experiencias cognitivas, afectivas, instrumentales y morales; depuradas, ordenadas y sistematizadas por las artes, los sistemas de pensamiento, la ciencia, las tecnologías, las tradiciones, etc." (Calvo et al., 1999, 21). Las emociones serían, por tanto, una característica de la cultura a la que todo ser humano llega y de la que se hace heredero. Sin embargo, unas páginas más adelante, estos mismos autores sostienen que "ser persona de criterio significa ser capaz de conducir la propia vida de acuerdo con criterios fundados en convicciones racionales y existenciales. En consecuencia, resulta despersonalizado todo cuanto se opone a la actuación de la voluntad libre, bien sea debido a factores externos (ambiente o moda, manipulación, adoctrinamiento), bien a factores internos (caprichos, pasiones)" (Calvo et al., 1999, 59). Así, si bien las pasiones se identifican como elementos clave de la cultura, no resultan relevantes como guías para la acción.

En la siguiente ponencia los profesores P. M. Pérez Alonso-Geta, C. Cánovas y E. Gervilla se refieren a los valores y las actitudes como competencias básicas de los alumnos, y basan su ponencia en el concepto de competencia emocional desarrollado por D. Goleman (1996). Defienden que «la competencia emocional puede 
entenderse como la capacidad que permite a la especie humana solucionar el problema de la vida" (Cánovas et al., 1999, 63). La inteligencia emocional "comprende las capacidades básicas como la percepción y canalización de la propia emoción o la comprensión de los sentimientos de los demás. Tiene su propio dinamismo y actúa constantemente sobre nuestro comportamiento y personalidad. Estas capacidades básicas, que nos permiten tener confianza en nosotros mismos o saber disfrutar de la relación con otras personas, se van formando durante la infancia" (Cánovas et al., 1999, 60). Este tipo de inteligencia se compone de cuatro parámetros básicos: la capacidad de entender y comprender emociones y sentimientos propios, la autoestima, la capacidad de gestionar y controlar los impulsos y situaciones afectivas y la capacidad de entender y comprender los sentimientos de los demás.

Por su parte, los profesores M. Puig, F. Bárcena, J. Escámez y P. Ortega trabajan "Las competencias morales básicas en la formación de los ciudadanos". En este contexto abordan las competencias sociomorales, conformadas por un conjunto de capacidades entre las que destacan: el autoconocimiento, que incluye la subcapacidad de "conseguir una mayor transparencia sobre cuáles son los propios sentimientos, deseos y motivaciones" (1999, 11); el conocimiento de los demás, esto es, «la capacidad de experimentar en sí mismo los sentimientos ajenos distinguiéndolos de los propios" (1999, 11); la comprensión, capacidad que permite eliminar la dependencia de la reflexión moral respecto de las situaciones concretas y contextuales, para lo que apela no sólo a la razón, sino también al sentimiento y a las emociones, a la benevolencia y al amor; las capacidades emocionales y de sensibilidad, ya que estas capacidades permiten "detectar los conflictos morales, sentirse concernido y afectado por ellos, captar valorativamente la realidad, y finalmente impulsar y motivar la conducta de los sujetos con gran eficacia" (1999, 11); y, finalmente, la toma de conciencia, que permite conceptualizar y regular los procesos cognitivos, conductuales y emocionales.

En el SITE del año 2000, titulado Educación y calidad de vida, encontramos dos ponencias que abordan la relación entre ser humano y emociones. La primera de ellas, presentada por los profesores J. M. Asensio, F. Bárcena y J. C. Mèlich, defiende un nuevo concepto de cognición más amplio que el de pensamiento, afirmando que incluye también "percepción, emoción y acción" (1998, 197). Reclaman la vinculación radical de la cognición con los estados afectivos; resaltan que, frente a la concepción dominante de la escisión entre razón y emoción y la primacía de la primera sobre la segunda en la evolución de la especie humana, tal radical unión ha desempeñado un papel fundamental en nuestra evolución, ya que "somos los descendientes de una especie que surgió trabada por unas relaciones afectivas", por lo que "cabe plantearse la razonable posibilidad de que el camino evolutivo que permitiera separarnos del resto de los primates fuera abierto, preponderantemente, por la "biología del amor", (Asensio et al., 2000, 93). La humanización del hombre radica en su capacidad para amar y en su desarrollo afectivo y moral. Y esto es así porque "para lo mejor o para lo peor somos conscientes de nuestras vivencias. 
Nadie como el hombre puede en este sentido gozar o sufrir de su existencia. De ahí la necesidad de un compromiso moral, de la emergencia de unos sentimientos fraternales que [...] están en los orígenes de lo humano" (2000, 94).

Por su parte, los profesores J. Trilla, A. Ayuste, T. Romañá y H. Fuentes presentan la ponencia titulada "Educación y calidad de vida. Las cosas, los otros y uno mismo". En ésta también reclaman un concepto más amplio de cognición, que incluye la emoción y la acción. Afirman con ello que "ya no es suficiente hablar de que el hombre es un ser racional, es mucho más que eso", ya que "admitimos que la racionalidad es nuestra mejor herramienta para pensar y hacer en el mundo, pero ¿lo serio no es acaso pensado desde el corazón?» (Ayuste et al., 2000, 164). Achacan el tardío reconocimiento de "ese fluir de sentimientos, pensamientos, instintos" a la dificultad de las herramientas epistemológicas del saber occidental para captar la ineludible "inefabilidad humana".

P. Ortega abría el site del año 2001, Conflicto, violencia y educación, con el anuncio de la crisis de la razón instrumental que había hecho creer al ser humano que la solución a sus problemas radicaba en la ciencia y la tecnología, dejando de lado los principios que regulan la relación entre los hombres. Estos principios conforman los valores morales que, para el autor, padecen el abandono y la marginación en la actual sociedad. Recuerda que "los valores se aprenden y se enseñan", añadiendo que "este tipo de aprendizaje exige, además, un clima de afecto. La apropiación del valor no es fruto de una simple operación de cálculo; interviene, en gran medida, la mediación del modelo que hace atractivo, sugerente el valor" (Ortega, 2001, 13).

A esta presentación sigue la ponencia "La crisis de la sociedad actual", de los profesores G. Jover, P. Casares, F. Gil y M. Payá, quienes retoman la crisis de la razón y el consecuente surgimiento de un nuevo sujeto, que, sin embargo, no se trata ya "de un yo desencarnado, de una pura razón, sino de sujetos reales aquí y ahora, con voz e historias, sufrimientos y deseos" (Casares et al., 2001, 31).

Un año después, en el Xxi site celebrado en Granada, serán los profesores J. García Carrasco, B. Gros y A. Ayuste los encargados de traer de nuevo a colación las emociones en la idea de ser humano que se asienta tras la reflexión pedagógica. En su ponencia "Sociedad-Red, educación e identidad" los autores critican el surgimiento de un nuevo mundo, en el que la interpretación de éste queda reducida a una vertiente tecnológica que rige la organización social, quedando "diluidas las necesidades primarias de unos sujetos que la evolución dispuso como seres vivos corporales, emocionales, lectores de mente, con pegajosidad biológica en el comportamiento relacional, sociales y cooperativos en la proximidad de la presencia" (Ayuste et al., 2002, 32).

El XXI siTE, celebrado en Sitges en el año 2003, explora Otros lenguajes en educación. A este respecto, los profesores X. Úcar, R. Cortada y M. C. Pereira profundizan en los lenguajes del arte y sus posibilidades para la formación integral. Para ello retoman los conceptos de Zubiri «inteligencia sintiente, voluntad tendente, sentimiento afectante" para referirse a la intrínseca unidad del ser humano y, como 
consecuencia, la necesidad de concebirlo como un todo integrado (Cortada et al., 2003, 109).

Tras ellos, y continuando con esta llamada a la concepción del ser humano como un todo, serán los profesores F. Bárcena, H. Tizio, J. Larrosa y J. M. Asensio quienes rescaten la importancia del cuerpo como lugar del ser humano. Un cuerpo que transita por la vida "a la búsqueda activa de otros cuerpos semejantes al nuestro sin los que sería imposible [...] acceder al mundo de los símbolos o al de los signos que cada cultura y grupo humano especifican" (Asensio et al., 2003, 135) y que se hace posible gracias al afecto. Las expresiones corporales son un elemento esencial en el desarrollo del habla como expresión del afecto buscado en los otros, convirtiéndose en el sustento de los estados emocionales, sobre los que a su vez se fundamentan el habla, la razón y cualquier relación comunicativa. Denuncian estos autores el olvido del que ha sido víctima el cuerpo en su tradicional oposición a la mente, jerarquización sobre la que se han construido los diversos significados e interpretaciones del conocimiento, la inteligencia, los sentimientos, el lenguaje o la conciencia, caracterizados todos ellos por la localización en "una mente ingrávida que conoce y nos representa en nuestras relaciones con el mundo más allá de nuestro propio cuerpo" $(2003,138)$.

En la siguiente y última ponencia de este Seminario, los profesores C. Naval, C. Sábada, X. Bringué y P. M. Pérez se refieren también al lenguaje como expresión de una tendencia previa del ser humano hacia los otros y a la búsqueda de afecto que se encuentra en la base de esta tendencia; el lenguaje no busca sólo mostrar, sino también "mover o conmover" (Bringué et al., 2003, 179).

En el xxiI Seminario se retoma la relación entre los valores y las emociones. En este caso serán los profesores F. Altarejos, M. Martínez, M. R. Buxarrais y A. Bernal quienes afirmen que "la vivencia del valor no es, en la mayoría de los casos, el resultado de una deliberación racional, sino de una apropiación que se mueve tanto en el ámbito racional como en el emotivo" (Altarejos et al., 2004, 125).

Llegamos con esto al año 2006, en el que la celebración del Seminario gira en torno a La vida emocional. Las emociones y la formación de la identidad humana. El título refleja la concepción de las emociones que se va fraguando progresivamente durante estos años, esto es, que el sistema emocional no constituye un elemento aislado de la racionalidad, sino que ambos se encuentran en relación permanente, de manera que las emociones se encuentran imbuidas de racionalidad, de la misma forma que la racionalidad se constituye en relación a la información proporcionada por el sistema emocional. Así, P. Ortega abre nuevamente el Seminario defendiendo que

la secular forma dual de concebir al ser humano, separando en él los dominios afectivos de los racionales, lo corporal de lo mental, carece ya de sentido. Y que las emociones, lejos de ser un permanente riesgo para nuestra racionalidad, contribuyen, las más de las veces, a hacerla más eficiente, a que sus juicios se adapten mejor a la experiencia vivida, cuando no a echarle una decisiva mano en la valoración de asuntos complejos que nos conciernen especialmente y que desbordan nuestras 
capacidades de cálculo y decisión. [...] Lo que se empieza a conocer acerca de los estados afectivos apunta en el sentido de destacar el hecho de que desempeñan un papel esencial en el desarrollo de nuestras capacidades de relación, de nuestra inteligencia e incluso de nuestra condición ética. El ser humano, lejos de hipotecar su racionalidad por la irrupción en su mente de unos u otros sentimientos, lo que hace con ellos es enriquecer su intuición, la agudeza de sus pensamientos, la humanidad de sus acciones. Toda la construcción de la identidad del sujeto está amalgamada de cognición y emoción (García Carrasco et al., 2006, 23).

El progresivo reconocimiento que las emociones van adquiriendo como configuradoras del carácter propiamente humano, junto con la dedicación de un Seminario a la reflexión en torno a este asunto, permiten abordar reflexiones más profundas en cuanto a la relación que el ser humano establece con lo que siente. Reflexiones que sitúan a las emociones en el centro de la experiencia:

Este es el nítido mensaje que nos transmiten [las emociones] a través del cuerpo: el de cómo me afecta lo que vivo, no cómo desearía cognitivamente que me influyera teniendo en cuenta unos u otros preceptos morales o propuestas educativas. Y conviene tomar en consideración esa cualidad de las emociones que nos sitúa permanentemente en la realidad de lo que sentimos con independencia de si ello es o no política, social o moralmente correcto (Acarín et al., 2006, 44).

Añaden que lo que sentimos puede no estar en la línea de lo que consideramos que está bien, incluso contradecirlo, lo que supone un pequeño apunte a la complejidad de la cuestión. En relación a esta complejidad señalan que "nada hay que temer pues de los estados afectivos, sino todo lo contrario, salvo los posibles errores en sus evaluaciones o una inadecuada respuesta a las mismas" (2006, 45). Así, si bien las emociones son una guía esencial en la construcción de la identidad y en la toma de decisiones, la información que nos aporten puede ser malinterpretada. Finalizan afirmando que

no parece que entre nuestras cualidades se encuentre la de que un determinado discurso verbal, por sí mismo, por su exclusiva racionalidad, nos mueva a variar conductas, afectos y opiniones por sí mismo. [...] Lo que sí pueden hacer, quizás, las "buenas razones" es mover nuestros afectos en el sentido que proponen si, como señala este autor [Maturana] estamos en condiciones de considerarlas abuenas" al participar en el sistema de valores en que se fundamentan $(2006,46)$.

"Se escamotee o se asuma, [...], los seres humanos caminan por donde el sentimiento les lleva" (Bernal et al., 2006,76), postulan, en la ponencia que sigue, los profesores J. García Carrasco, R. Canal, A. Bernal y A. Martín Víctor. Entre otros, el camino al que estos sentimientos conducen es el del ejercicio de la ciudadanía. "Al cabo nuestros conceptos democráticos y las imágenes que guían nuestra ciudadanía quedan cojos cuando se intentan reconstruir en una historia que olvida sus débitos sentimentales. [...] De cosas tales como la naturaleza humana o la dignidad 
humana pudimos comenzar a hablar en su día porque nuestra educación sentimental nos permitió sentirlas cercanas y reales" $(2006,46)$.

Si hasta ahora se habían vinculado las emociones con el compromiso moral, estos profesores resaltan un aspecto más concreto de esta vinculación: las emociones y el ejercicio cívico. Esta relación anida en el seno de la vulnerabilidad humana, a la que los profesores aluden cuando refieren que "la complejidad de competencias de que dispone la mente de un ser humano deja abierta la posibilidad al mal paso, al mal trago, a la imperfección, a tener que afrontar la desdicha, el dolor y la sombra del padecimiento emocional" $(2006,84)^{5}$.

Las referencias a la vida emocional se retoman en el siTe de 2008, celebrado en torno a Educación y ciudadanía, tema que destaca y retoma lo que ya en el 2006 apuntaban los profesores García Garrido y otros. Será en este caso la profesora P. M. ${ }^{a}$ Pérez Alonso-Geta (2008) quien, en su presentación, señale la presencia de las emociones en las relaciones de convivencia con los demás, en la que éstas median y son la condición básica para la relación, posibilitando la inferencia de sus pensamientos, expectativas y también sentimientos. Esta capacidad de inferir y, también, comprender los sentimientos ajenos es el elemento esencial de la solidaridad que, según esta autora, se compone primera y fundamentalmente de compasión, entendida como "la capacidad emocional de comprender sentimientos y emociones ajenos, desde la perspectiva del otro" $(2008,96)$.

A continuación, los profesores F. Gil, G. Jover, C. Naval y D. Reyero aluden al papel que desempeñan las emociones no sólo en el ámbito moral, sino en la expresión más concreta de éste que es la ciudadanía, apelando al ciudadano disidente como aquel "sentimentalmente prudente, en el sentido de que no obstaculiza su proyecto de vida, las pasiones de sus vínculos de adscripción, sino que mantiene en cada circunstancia un afecto y un juicio ponderado" (Gil et al., 2008, 60).

\subsection{Consideraciones sobre el concepto de educación}

Finalizando con esto las referencias a las emociones en la aproximación a la definición del ser humano, abordaremos a continuación las referencias relativas al concepto de educación.

Retomamos, por tanto, el siTE celebrado en el año 1994 en torno a la Antropología de la educación. Los profesores G. Janer y A. J. Colom afirman que esta disciplina se presenta como «una ayuda de extraordinaria utilidad en la consolidación de la educación entendida tecnológicamente, o sea, centrada en el logro de acciones eficaces a fin de cumplimentar lo más fehacientemente posible el logro

5. Este año se celebraba el xxv aniversario del site; la organización del Seminario decidió reforzar la importancia del tema publicando, de manera paralela, un número monográfico de la Revista Interuniversitaria de Teoría de la Educación, vol. 18, bajo el lema "Educación y emociones". 
educativo e instructivo que se propone" (Colom y Janer, 1995, 94). Por su parte, los profesores J. A. Jordán, F. Etxeberría y J. Sarramona, que, como se ha visto anteriormente, hacían hincapié en el papel de las emociones en la construcción de la identidad, definen al hombre educado como "alguien capaz de enraizarse consciente y profundamente en un grupo cultural, así como de realizar, a través de profundas transvaloraciones, un movimiento dialéctico cognitivo/actitudinal de "salidas y retornos", de apertura y de vuelta a la identidad cultural de base para -de ese modo- conocerla, enriquecerla y mejorarla” (Etxeberría et al., 1995, 123).

El edificio conceptual del discurso educacional, que se contiene en muchos textos pedagógicos, está fundamentalmente organizado por los convoyes semánticos de aprendizaje y enseñanza: patrones de comportamientos, conocimientos, competencias, apreciaciones y concepciones del mundo que se enseñan y se aprenden. [...] El afecto, la sensibilidad, la afectividad estaban incluidos, pero al tomar el corpus discursivo entre las manos estos aspectos han sido los gelatinosos que se escapaban entre los dedos, los que no sostenían el tamiz analítico porque, en el fondo, el afecto se muestra pero no se enseña, la afectividad se induce pero no se instruye, la emoción se genera pero no se aprende (Caride et al., 1995, 152).

Con estas palabras introducen los profesores J. García Carrasco, J. L. Núñez y A. Caride el contenido de su ponencia, también mencionada anteriormente y que versa explícita y directamente sobre la educación afectiva. Denuncian los ponentes que la emoción ha sido "la cenicienta" de las ciencias humanas, entre ellas también la educación, y señalan la necesidad de incluir el desarrollo emocional dentro de las finalidades educativas, de la misma manera que se consideran el intelectual y el ético.

Si seguimos manteniendo que la pedagogía es el ámbito de conocimiento de los procesos educacionales cuya intención es el dar y proporcionar explicaciones y plantear cursos de acción intencionales para agentes educativos, también debe comprometerse con esos ámbitos en los que muchos individuos dilucidan sus compromisos personales, sus compromisos morales, su participación y disfrute de muchos bienes culturales y hasta la estructura-desestructura de su personalidad. No se trata por lo tanto de construir la acción pedagógica desde las emociones, los sentimientos o los afectos, sino de dar razón de ellos en el momento de decidir sobre la acción razonable (Caride et al., 1995, 154).

Y esto ha de ser así porque las decisiones que toman los sujetos de la educación se fundamentan y explican en razón del gusto-digusto, afecto-repulsa, atracción-rechazo, que generan en ellos las acciones, situaciones, etc.

También J. M. Esteve, J. M. Puig y T. Romañá, un año después, destacan el papel de los grupos primarios en los procesos formativos.

La importancia que tiene la percepción que cada sujeto se hace del ambiente del grupo por encima de la supuesta influencia que pueda ejercer su realidad "objetiva.. [...] El significado personal y social que tienen los fenómenos grupales, el 
matiz emocional con que se viven, la capacidad motivadora que desatan, e incluso las percepciones imaginarias e irreales que desencadenan dependen de cómo cada sujeto sea capaz de reconocer la situación. Este es un dinamismo a la vez personal, social y cultural cuya influencia en los procesos formativos resulta ineludible (Esteve et al., 1999, 74).

Sin embargo, es necesario tener presente que la referencia a los grupos primarios señala, fundamentalmente, a la familia. Así lo afirman los ponentes, quienes afirman que la primera e ineludible tarea de los padres es formar actitudes radicales. Estas actitudes radicales de apertura y de clausura configuran el proceso formativo de la personalidad y facilitan o dificultan su desarrollo. Finalmente, estos profesores señalan el influjo de los grupos primarios en la adopción de roles, proceso de aprendizaje complejo y que guarda una estrecha relación con la formación moral. "El proceso de adquisición de roles supone un nivel de descentración tal que permita ponerse en el lugar de otras personas y realizar inferencias acerca de sus estados y comportamientos previsibles. Reconocer las ideas y sentimientos ajenos y prever sus comportamientos es una competencia social básica" (1999, 84).

Continuando con el siguiente Seminario, celebrado en 1996 y que llevó por título Tecnologías y formación permanente, P. Aznar, A. Barrón y B. Gargallo defienden un enfoque tecnológico de la educación desde el que la mente humana actúa como un procesador de información que no sólo procesa datos, sino que los maneja flexiblemente, relacionándolos con las experiencias cognitivas y afectivas anteriores, confiriendo a la nueva información un significado coherente. Continúan identificando los procesos cognitivo-afectivos como uno de los procesos principales que el sujeto pone en marcha para aprender. Sin embargo, al detallar los procesos que pertenecen a este ámbito señalan la atención, percepción, codificación y almacenamiento, etc., sin hacer mención a elementos emocionales. Sí se refieren explícitamente a ellos cuando reclaman la necesidad de un cambio de rol de la Tecnología educativa, desde la focalización en la enseñanza hacia el aprendizaje. Este cambio de rol supone "un cambio desde la atención puesta casi únicamente en los efectos de los factores cognitivos, hacia la consideración de los efectos afectivos en la cognición en general y el impacto específico de las variables motivacionales sobre el aprendizaje" (Aznar et al., 1997, 135).

El año siguiente el SITE gira en torno a La formación docente. El profesor F. Altarejos defiende la necesidad de la templanza en los docentes, "una armonía interior sólida que impida desmesuras en la sensibilidad subjetiva" (1998, 106). También a los docentes, en este caso universitarios, se refieren M. Martínez, B. Gros y T. Romañá el año siguiente, en 1998, en el que La educación universitaria: nuevos retos educativos y tecnológicos es la temática escogida. El buen profesor es quien es capaz de transmitir a sus alumnos "pasión por el conocimiento, por la búsqueda de la verdad, por la apertura al disenso". Continúan afirmando que, en el aula y una vez tomadas las decisiones acerca del contenido y la metodología de trabajo, la comunicación deviene la cuestión principal. En relación a ella afirman que los 
docentes deben transmitir emociones, no sólo hechos, y que deben crear un clima favorable para la comunicación, en el que "el sentimiento sea mutuo" (1998, 44).

Y con estas menciones retomamos nuevamente el año 1999, las competencias básicas y la ponencia de los profesores J. Vera, A. García del Dujo, P. Calvo y B. Gargallo (Calvo et al., 1999) acerca de los criterios de selección del currículum. Esta selección ha de tener en cuenta, en relación al sujeto que aprende y además de otros factores, su desarrollo emocional, racional, moral, físico y cognitivo.

A continuación, P. M. Pérez Alonso-Geta, C. Cánovas y E. Gervilla consideran que profundizar en la competencia emocional del alumnado es esencial en la educación obligatoria. Las emociones se encuentran presentes en la naturaleza humana, pero es la cultura la que determina y concreta esta posibilidad en lo que los sujetos llegan a ser en su seno.

En el seno de una determinada cultura se aprende a manifestar o controlar sentimientos y emociones de acuerdo a los significados, valores y pautas culturales del grupo. Nuestro equipamiento biológico nos permitirá vivir una serie de vidas afectivas posibles, pero nacemos en una cultura y acabamos viviendo una sola vida cultural. Sin embargo, ser humano significa algo más que sentir y actuar como miembro de un grupo cultural, hay diferentes modos "individuales" de sentir y ser de ese grupo. Este proceso de individualización personal se desarrolla a través de la educación. Gracias a la educación se lleva a cabo la modulación cultural de lo biológico. El ambiente modula la competencia o incompetencia emocional (Cánovas et al., 1999, 60).

La competencia emocional se identifica con la autorregulación, el autocontrol y la canalización de las emociones, que se adquieren a través de la educación.

Retomemos el site celebrado en el año 2000 bajo el tema de Educación y calidad de vida. Los profesores A. J. Colom, P. Pérez Alonso-Geta y G. Vázquez Gómez abren el seminario afirmando que "educar para la vida es educar para la vida del hombre, en su sentido personal (moral, afectivo, intelectual, cultural y físico), social (desarrollo convivencial y social), laboral e incluso político. Así, una educación integral e integradora debe incluir entre sus enseñanzas el aprendizaje del manejo de los afectos y la competencia emocional". Para ello se requiere "unificar múltiples pedagogías que ahora se orientan con independencia las unas de las otras" (Colom et al., 2001, 35), entre las que se encuentran la educación cívico-social, la educación moral, la educación para la paz, la educación para la igualdad de sexos, etc.

Los profesores J. M. Asensio, F. Bárcena y J. C. Mèlich defienden que los cambios culturales pueden desestabilizar la manifestación de las tendencias afectivas, haciendo más hincapié en otros aspectos de la realidad humana, y consideran a la educación la responsable de estabilizar "lo humano de nuestro ser y de contribuir con ello a generar unas formas de convivencia que armonicen con nuestras más íntimas expectativas" (Asensio et al., 2001, 94). Destacan que "la conciencia del educador es -como dice Jorge Larrosa- mucho menos una conciencia formada por lo racional y lo funcional que por la sensibilidad y las emociones, o sea, por su capacidad de dejarse afectar por el otro y padecer con él” (2001, 99). 
Continuando con esta concepción holística del ser humano, así como de la propia educación como proceso de humanización, los profesores J. Trilla, A. Ayuste, T. Romañá y H. Fuentes defienden la recuperación por parte de la educación de "una mayor autoconsciencia de nuestros deseos, necesidades, sentires y experiencias" (Ayuste et al., 2000, 144).

El sITE celebrado en 2001 se abre con la ya mencionada ponencia de los profesores G. Jover, P. Casares, F. Gil y M. Payá, quienes defienden que "se tendría que reclamar más espacio para las emociones, desintelectualizar algunas prácticas y personalizar más, volviendo a relaciones educativas más próximas" (Casares et al., 2001, 39). La concreción educativa de este reclamo conlleva ayudar a los alumnos y alumnas a reconocer tanto sus propias emociones como las de los demás.

Sigue a esta ponencia la presentada por los profesores F. Etxeberría, J. M. Esteve y J. A. Jordán (Esteve et al., 2001), quienes también reclaman la necesidad de la educación emocional, tanto desde la perspectiva de la identificación de las emociones, como desde su expresión y recepción. Destacan el papel del profesorado, a quien atribuyen la tarea de dar más importancia a los aspectos emocionales y afectivos de la formación en cada una de sus áreas de trabajo. También los últimos ponentes de este Seminario, J. Escámez, R. García, M. A. Sales y J. M. Rodríguez, aluden al papel y la figura del profesor para la educación de las emociones (Escámez et al., 2001, 225).

En la presentación del xxII SITE celebrado en Sitges en el año 2003, podemos leer: "En este nuevo encuentro, se trata de reflexionar sobre la apertura de la educación hacia otras escenas formativas ("otras" frente a los sesgos habituales en teoría de la educación) que incluyen necesariamente dimensiones físicas, sensoriales, afectivo-emocionales e imaginativas... además de cognitivas" (Martínez, Romañá, 2003, 7).

Esta apertura anunciada pasa, según los profesores X. Úcar, R. Cortada y M. C. Pereira, por crear espacios teóricos donde no sólo la ciencia o la técnica, sino también el arte, sean considerados. Denuncian los profesores la estigmatización de que han sido objeto determinados ámbitos curriculares, fundada en una tradición que «ubicaba la ciencia, la cultura y lo formativo en el ámbito de lo cognoscible, lo manejable y, por tanto, de lo que proporciona seguridad. A lo demás se le tildaba de irracional o, simplemente, se desprestigiaba acusándolo de estar mediatizado por la subjetividad o por la afectividad" (Cortada et al., 2003, 75). Las tradicionalmente desprestigiadas artes, especialmente el teatro y el cine, ofrecen múltiples posibilidades para la formación integral, con especial referencia al desarrollo y la formación de las emociones.

P. Ortega, en su ya mencionada presentación del xxIv Seminario, reconoce que

El estudio de los sentimientos, o mejor dicho, la educación de los sentimientos no ha sido un tema preferente entre los pedagogos españoles. Nuestra preocupación se ha centrado más en el desarrollo de las competencias intelectuales, dejando en un segundo lugar aquellos componentes de la educación como los sentimientos, 
las emociones o los afectos. Ello ha dado lugar a un fuerte desequilibrio entre lo cognitivo y lo emocional en los contenidos curriculares y en las propuestas educativas. En las últimas décadas, sin embargo, se ha rescatado la idea clásica de la educación que no se limita al aprendizaje de conocimientos, sino que incorpora los sentimientos como contenidos básicos de toda persona educada (AA.VV., 2006, 16).

Retoman esta declaración de intenciones J. M. Asensio, N. Acarín y C. Romero, quienes se muestran desconcertados ante la falta de atención que la investigación pedagógica presta al componente emocional. Abordan en su ponencia la relación entre las emociones y el compromiso moral, afirmando que

no es a través de la razón como las personas deciden profesar una u otra religión, hacerse más o menos solidarios o formar parte de colectivos que se dediquen a tareas humanitarias, sino de los afectos generados a través de la experiencia o de su íntimo pensar. De un reflexionar que, eso sí, puede movilizar sus sentimientos en esas direcciones, pero no conseguirlo directamente por su exclusivo poder de inducción lógico (Acarín et al., 2006, 58).

Los profesores J. García Carrasco, R. Canal, A. Bernal y A. Martín Víctor refieren el componente emocional desde una perspectiva no mencionada con anterioridad, esto es, las disfunciones y las discapacidades. Defienden que el sujeto de la pedagogía ha sido siempre el sujeto "normal", regido por un funcionamiento apropiado de la mente, con relaciones regulares con el mundo. "Parece el funcionamiento normal, la construcción, el perfeccionamiento, la fuente principal de inspiración" (Bernal et al., 2006, 84). Frente a esta concepción de la pedagogía como actividad de perfeccionamiento, entendido éste como llegar a ser lo que no somos, García Carrasco y otros proponen la misión de la pedagogía como aprendizaje de lo que somos, como asunción de la vulnerabilidad inherente a nuestra condición. "Bucear en el padecimiento emocional, desde una actitud de biofilia, tiene el beneficio de comprobar con sorpresa que tal padecimiento se encuentra generalizado, en mayor o menor medida; nos propone la imperfección, desde el principio y por principio, como perteneciente con todo rigor a la condición humana" (2006, 84). Éste, el sujeto que padece, que siente y encuentra entre sus sentires el sufrimiento, éste ha de ser el sujeto de la pedagogía, dado que es el sujeto de la vida.

En este mismo site los profesores L. Núñez Cubero, R. Bisquerra, J. González Monteagudo y M. C. Gutiérrez vuelven a reclamar la necesidad de la educación emocional, conducente al aumento del bienestar, tanto de la persona como de la sociedad en su conjunto. Para llevar a cabo esta educación consideran también necesaria la investigación pedagógica en este ámbito, ya que "conocer las propias emociones, la relación que éstas tienen con nuestros pensamientos y comportamientos, debería ser uno de los objetivos de la educación" (Bisquerra, 2006, 188). 
MIRIAM PRIETO EGIDO

\section{CONCluSiones}

En nuestros días es habitual escuchar hablar de la dupla razón-pasión, así como la afirmación de que desde Platón hasta nuestros días se ha atendido, en todas las facetas del ser humano, sólo a la primera de ellas, quedando las pasiones relegadas al olvido. Si bien es cierto que siempre ha existido una jerarquización de ambas, situándose a la razón por encima de las pasiones (lo que se puede comprobar en la concepción del ser humano como ser primera y eminentemente racional), también es cierto que la mención a las pasiones es una constante más o menos evidente en la historia del pensamiento acerca del hombre. Parece como si el ser humano, al tratar de definirse a sí mismo, intuyese o supiese de la existencia de un elemento no del todo incluido o expresado en el término "racional". Un componente que tal vez no se haya sabido definir o nombrar, un elemento "gelatinoso" (Caride et al., 1995, 155) que no se ha podido retener entre las manos de la explicación, quizás tampoco articular en relación a la racionalidad, pero que no se ha podido excluir definitivamente al tratar de hablar de quién somos. Así, en ocasiones vagamente, en otras despectivamente, la mención a las emociones es una constante en las distintas manifestaciones del ser humano a lo largo de la historia. Constante que, como muestra esta revisión, encontramos también en la Teoría de la Educación española elaborada en el site en las últimas décadas.

Si bien las ponencias que abordan de manera explícita y central la temática afectiva se limitan a seis, la referencia a la vida sentimental es una constante que aparece repetidamente al abordar cuestiones tan diversas como la formación de la identidad, la formación docente, la comunicación educativa o las competencias básicas del alumno. De hecho, si revisamos las menciones arriba expuestas a la luz de los ámbitos de estudio de la Teoría de la Educación ${ }^{6}$ encontramos que todos se encuentran aludidos. No se trata, por tanto, de un interés puntual o personal ${ }^{7}$, sino un punto de paso necesario e ineludible cuando se aborda la reflexión acerca de la educación y los sujetos que de ella participan.

La necesidad de profundización y estudio de la vida emocional desde la Teoría de la Educación se ratifica también en la evolución apreciada en esta revisión acerca de la definición de ser humano que, sin embargo, no se ha seguido de una evolución de las mismas dimensiones en la concepción de la educación. De la

6. Epistemología pedagógica, procesos educacionales primarios, relaciones mente-cuerpo, pedagogía de los procesos cognitivos, pedagogía del comportamiento afectivo, pedagogía del comportamiento moral, la educación y su entorno, política educativa y pedagogía de la comunicación (BARRón et al., 1995).

7. De los 96 miembros del site que se registran en 2003 , 48 intervienen en las ponencias en las que se hace alusión a la vivencia sentimental. El listado de los profesores miembros puede consultarse en HERNÁNDEZ Prados (2003, 299-302), en OrTega (2003, 239-355). Sin embargo, desde ese año nuevos profesores han entrado a formar parte del siTe. Concretamente, son 13 los profesores autores de las ponencias que refieren en algún momento el aparato emocional y que no aparecen en el listado mencionado. 
tradicional definición del ser humano como ser racional pasamos a la consideración del hombre como el ser que piensa, siente y actúa, manifestaciones íntimamente ligadas entre sí y que no pueden pensarse aisladamente. Esta nueva concepción no supone un mero cambio, sino una forma diferente de hablar de quienes somos, un nuevo lenguaje que pone el acento en la fragilidad y la vulnerabilidad, en las fronteras de nuestra propia identidad. Este nuevo lenguaje sólo parcialmente se refleja en la concepción de la educación y en su relación con el sistema emocional, pues éste se concibe como un añadido a las finalidades cognitivas y morales de la educación, sin profundizar en la relación que guarda con ellas ${ }^{8}$.

\section{REFERENCIAS BIBLIOGRÁFICAS}

AA.Vv. (1983) Teoría de la Educación. (El problema de la educación I). Murcia, Límites.

- (1985) Condicionamientos sociopolíticos de la Educación. Barcelona, CEAC.

- (1986) Tecnología y Educación. Barcelona, cEAc.

- (2006) La vida emocional. Las emociones y la formación de la identidad humana. Barcelona, Ariel.

Acarín, N.; Asensio, J. M. y Romero, C. (2006) Emociones, desarrollo humano y relaciones educativas, en AA.VV. La vida emocional. Las emociones y la formación de la identidad humana. Barcelona, Ariel, 23-68.

Altarejos, F.; Bernal, A.; Buxarrais, M. R. y Martínez Martín, M. (2004) Familia, valores y educación, en Santos Rego, M. A. y Touriñán, J. M. (eds.) Familia, Educación y Sociedad Civil. Santiago de Compostela, Universidade de Santiago de Compostela, 89-136.

Asensio, J. M.; Bárcena, F.; Larrosa, J. y Tizio, H. (2003) El lenguaje del cuerpo. Políticas y poéticas del cuerpo en educación, en Romañé, M. T. y MarTínez, M. (eds.) Otros lenguajes en educación. Barcelona, ICE, 122-172.

Asensio, J. M.; BÁrcena, F. y MèLICH, J. C. (2000) Educación, vida humana y biopoder, en VÁzquez Gómez, G. (ed.) Educación y calidad de vida. Madrid, Complutense, 81-115.

Ayuste, A.; Fuentes, H.; Romañá, T. y Trilla, J. (2000) Educación y calidad de vida. Las cosas, los otros y uno mismo, en Vázquez, G. (ed.) Educación y calidad de vida. Madrid, Complutense, 117-167.

Ayuste, A.; García Carrasco, J. y Gros, B. (2002) Sociedad-Red, educación e identidad, en Gervilla, E. (coord.) Globalización, Inmigración y Educación. Granada, Diputación Provincial de Granada, 27-91.

Aznar, P.; Barrón, A. y Gargallo, B. (1997) Tecnología educativa y procesos de aprendizaje, en Barroso, C. y Gallardo, M. (coords.) Tecnologías y formación permanente. Tenerife, Universidad de La Laguna, 111-168.

Bárcena, F.; Escámez, J.; Ortega, P. y Puig, J. M. (1999) Las competencias morales básicas en la formación de los ciudadanos, p. 6. Se puede consultar en la página web del site: http://www.ucm.es/info/site/site18.html. Descargado el 21 de noviembre de 2010.

8. La referencia a los textos del Seminario así como las adendas presentadas y, en algunos casos, el texto completo, pueden consultarse en la página del Seminario: http://www.ucm.es/info/site/. 
Barrón, A.; García Carrasco, J.; García del Dujo, Á. y González Sánchez, M. (1992) La Teoría de la Educación en la actividad académica española. Análisis de un indicador. Teoría de la Educación, 4, 41-52.

Bernal, A.; Canal, R.; García Carrasco, J. y Martín, V. (2006) El sistema emocional y la circunstancia pedagógica, en AA.VV. La vida emocional. Las emociones y la formación de la identidad humana. Barcelona, Ariel, 71-167.

Bisquerra, R.; González Monteagudo, J.; Gutiérrez, M. C. y Núñez, L. (2006) Emociones y educación: Una perspectiva pedagógica, en AA.VV. La vida emocional. Las emociones y la formación de la identidad humana. Barcelona, Ariel, 171-196.

Bringué, X.; Naval, C.; Pérez Alonso-Geta, P. M. y SÁbada, C. (2003) Los lenguajes de las pantallas. Impacto en las relaciones sociales de los jóvenes y retos educativos, en Romañá, M. T. y Martínez, M. (eds.) Otros lenguajes en educación. Barcelona, ICE, 177-227.

BuXARrais, M. R. y MARTínez, M. (1996) Educación y modelos de aprendizaje social, en EsCÁMEz, J. (coord.) Acción educativa y comunicación social. Valencia, Tirant lo Blanch, 17-67.

Calvo, P.; García del Dujo, Á.; Gargallo, P. y Vera, J. (1999) Criterios de selección del currículum. Teoría de la Educación. Revista Interuniversitaria, 11, 13-52.

Cánovas, C.; Gervilla, E. y Pérez Alonso-Geta, P. M. (1999) Valores, actitudes y competencias básicas del alumno en la enseñanza obligatoria. Teoría de la Educación, 11, 53-83.

Caride, A.; García Carrasco, J. y Núñez Cubero, J. (1995) Educación afectiva y de la sensibilidad, en Noguera, J. (ed.) Cuestiones de Antropología de la Educación. Barcelona, CEAC, 151-192.

Casares, P.; Gil, F.; Jover, G. y Payá, M. (2001) La crisis de la sociedad actual, en Ortega, P. (ed.) Conflicto, violencia y educación. Murcia, Cajamurcia, 25-75.

Castillejo, J. L. (1988) Comunicación y construcción humana, en SarRamona, J. Comunicación y Educación. Barcelona, CEAC, pp. 45-62.

Colom, A. J. (ed.) (1994) Política y planificación educativa. Sevilla, Preu-Spínola.

- (2003) Lo político y lo comunitario en la Teoría de la Educación española. Una revisión, en Ortega, P. P. (ed.) Teoría de la Educación, ayer y hoy. Murcia, Selegráfica, 109-158.

Colom, A. J. y Janer, G. (1995) El modelo cultural en la construcción de la Antropología de la Educación, en Noguera, J. (ed.) Cuestiones de Antropología de la Educación. Barcelona, CEAC, 63-94.

Colom, J. A.; Pérez Alonso-Geta, P. M. y Vázquez Gómez, G. (2001) Calidad de vida, ambiente y educación, en VÁzQuez, G. (ed.) Educación y calidad de vida. Madrid, Complutense, $1-37$.

Cortada, R.; Pereira, M. C. y Úcar, X. (2003) Los lenguajes de las artes: escenas y escenarios en educación, en Romañá, M. T. y Martínez, M. (eds.) Otros lenguajes en educación. Barcelona, ICE, 73-116.

Damasio, A. (1994) Descartes' error: emotions, reason, and the human brain. New York, G. P. Putnam's Sons.

EsCÁmEZ, J. (1983) Educación y normatividad, en AA.Vv. Teoría de la Educación. (El problema de la educación I). Murcia, Límites, 65-77.

- (1985) Marginación socioeconómica y educación, en AA.vv. (1985) Condicionamientos sociopolíticos de la Educación. Barcelona, CEAC, 111-126.

- (1986) Los valores en la pedagogía de la intervención, en AA.Vv. Tecnología y Educación. Barcelona, CEAC, 157-173. 
Escámez, J. y Alonso-Geta, P. M. (1992) Planificación de programas de educación no formal, en Sarramona, J. La educación no formal. Barcelona, CEAC, 75-90.

Escámez, J.; García, R.; Rodríguez, J. M. y Sales, M. A. (2001) Educación y conflictos en la escuela. Propuestas educativas, en Ortega, P. (ed.) Conflicto, violencia y educación. Murcia, Cajamurcia, 209-235.

Esteve, J. M.; Etxeberría, F. y Jordán, J. A. (2001) La escuela y la crisis social, en Ortega, P. (ed.) Conflicto, violencia y educación. Murcia, Cajamurcia, 79-155.

Esteve, J. M.; Puig, J. M. y Romañé, T. (1996) La educación en los grupos primarios, en EscáMEZ, J. Acción educativa y comunicación social. Valencia, Tirant lo Blanch, 69-111.

Etxeberría, F.; Jordán, J. A. y Sarramona, J. (1995) Identidad cultural y educación, en una sociedad global, en Noguera, J. (ed.) Cuestiones de Antropología de la Educación. Barcelona, CEAC, 97-131.

EtXeberría, F.; Trilla, J. y Úcar, X. (1992) Educación no formal y políticas culturales, en SARRAMONA, J. La educación no formal. Barcelona, CEAC, 243-278.

Ferrández, A. y Sarramona, J. (1984) La educación. Constantes y problemática actual. Barcelona, CEAC.

Fullat, O. (1987) Fundamentación política del currículum, en Sarramona, J. (ed.) Currículum y Educación. Barcelona, CEAC, 47-58.

Fullat, O. y Mèlich, J. C. (1995) Del animal insuficiente a los valores, en Noguera, J. (ed.) Cuestiones de Antropología de la Educación. Barcelona, CEAC, 19-60.

Gil, F.; Jover, G.; Naval, C. y ReYero, D. (2008) El diseño curricular de la educación para la ciudadanía y los derechos humanos: debate social y análisis pedagógico, en VALDIvieso, S. y Almeida, A. S. (eds.) Educación y ciudadanía. Las Palmas de Gran Canaria, Anroart, 19-80.

Goleman, D. (1996) Inteligencia emocional. Barcelona, Kairós.

Hernández Prados, M. A. (2003) El Seminario Interuniversitario de Teoría de la Educación, por dentro, en Ortega, P. (ed.) Teoría de la Educación, ayer y hoy. Murcia, Selegráfica, 239-355.

IbáÑEz-MarTín, J. A. (1987) El problema del currículo: un primer acercamiento desde la Filosofía de la Educación, en Sarramona, J. (ed.) Currículum y Educación. Barcelona, CEAC, 13-24.

López-Barajas, E. (1988) Comunicación educador-educando (presencial y a distancia), en Sarramona, J. (ed.) Comunicación y Educación. Barcelona, CEAC, 65-79.

MaRín, R. (1983) La educación como optimización del hombre, en AA.VV. Teoría de la Educación I. (El problema de la Educación). Murcia, Límites, 109-123.

Martínez, M. y Romañá, T. (2003) Otros lenguajes en educación. Barcelona, ICE.

Medina, R. y Rodríguez, T. (1988) Fundamentación antropológica de la comunicación interpersonal, en Sarramona, J. Comunicación y Educación. Barcelona, CEAC, 15-41.

Naval, C. (2008) Teoría de la Educación. Un análisis epistemológico. Barañáin, Eunsa.

Ortega, P. (ed.) Conflicto, violencia y educación. Murcia, Cajamurcia, 209-235.

Pérez-Alonso Geta, P. M. (2008) La formación de la ciudadanía. La educación para la ciudadanía, en Valdivieso, S. y Almeida, A. S. (eds.) Educación y ciudadanía. Las Palmas de Gran Canaria, Anroart, 11-18.

SANVICENS, A. (1985) Educación y medios de comunicación social, en AA.Vv. Condicionamientos sociopolíticos de la Educación. Barcelona, CEAC.

Sarramona, J. (ed.) (1987) Currículum y Educación. Barcelona, Ceac.

- (1988) Comunicación y Educación. Barcelona, CEAC. 